SUPPORTING INFORMATION

\title{
Radiometal-containing aryl diazonium salts for chemoselective bioconjugation of tyrosine residues
}

\author{
Samantha Leier, ${ }^{1}$ Susan Richter, ${ }^{1}$ Ralf Bergmann, ${ }^{2}$ Melinda Wuest, ${ }^{1,5}$ Frank Wuest ${ }^{1,3,4,5,{ }^{*}}$
}

${ }^{1}$ Department of Oncology, University of Alberta, Edmonton, Canada

${ }^{2}$ Helmholtz-Zentrum Dresden-Rossendorf, Institute of Radiopharmaceutical Cancer Research, Dresden, Germany

${ }^{3}$ Faculty of Pharmacy and Pharmaceutical Sciences, University of Alberta, Edmonton, Canada

${ }^{4}$ Department of Chemistry, University of Alberta, Edmonton, Canada

${ }^{5}$ Cancer Research Institute of Northern Alberta, University of Alberta, Edmonton, Canada

*Corresponding author. Tel.: +1-780-989-8150; fax: +1-780-432-8483; Email: wuest@ualberta.ca 


\section{Table of Contents}

1. Reaction conditions using pre-conjugation versus post-conjugation labeling .........................................S3

Table S1 Reaction conditions tested for azo coupling of L-tyrosine with diazonium salt of $p-\mathrm{NH}_{2}-\mathrm{Bn}-\mathrm{NOTA}$.........S3

Table S2 Reaction conditions tested for azo coupling of L-tyrosine with diazonium salt of 3-fluoro-aniline ............S3

Table S3 Reaction conditions tested for azo coupling of L-tyrosine with diazonium salt of nat $\mathrm{Cu}$ or ${ }^{\text {nat }} \mathrm{Ga-labeled} p$ -

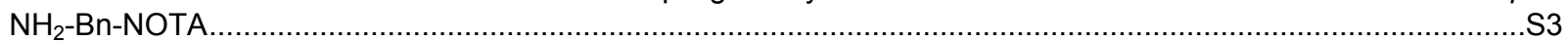

Table S4 Reaction conditions tested for azo coupling of neurotensin NT(8-13) with diazonium salt of $p-\mathrm{NH}_{2}-\mathrm{Bn}$ NOTA and reaction conditions tested for azo coupling of neurotensin NT(8-13) with diazonium salt of ${ }^{\text {nat }} \mathrm{Cu}$ or

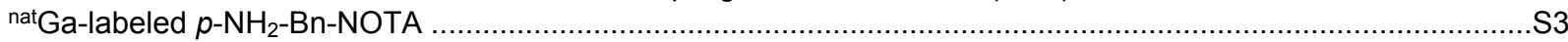

2. LC-MS spectra

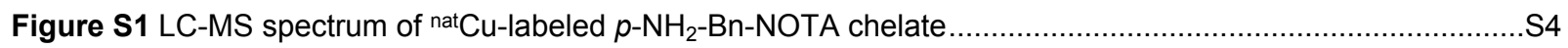

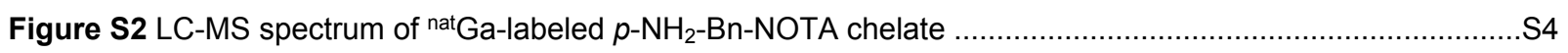

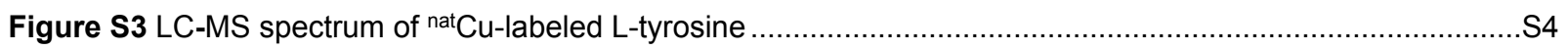

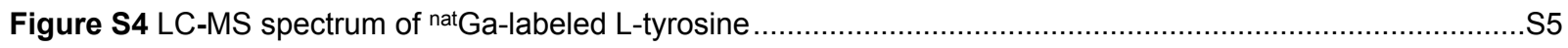

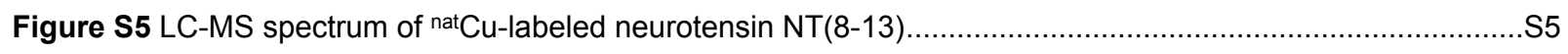

Figure S6 LC-MS spectrum of natGa-labeled neurotensin NT(8-13) ............................................................... 5

3. HPLC traces

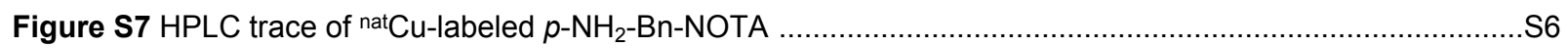

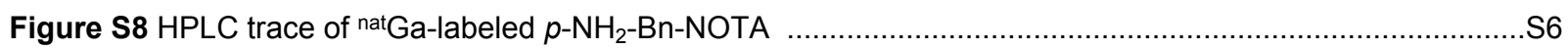

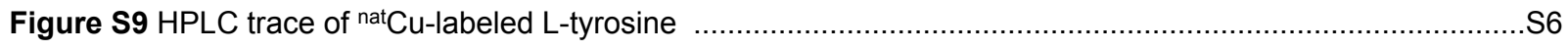

Figure S10 HPLC trace of natGa-labeled L-tyrosine .............................................................................

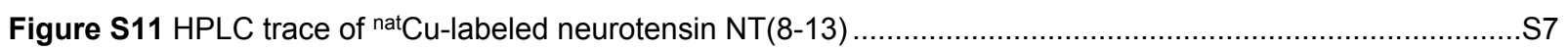

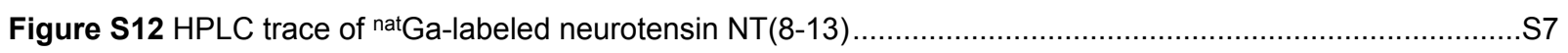

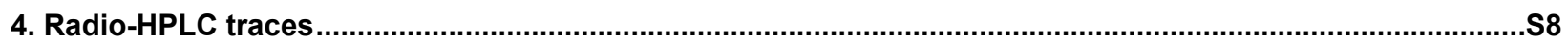

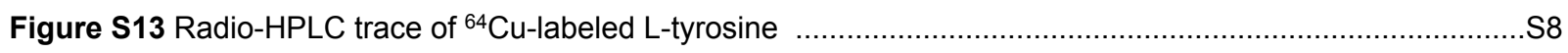

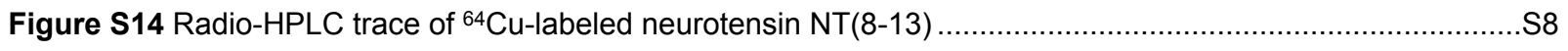

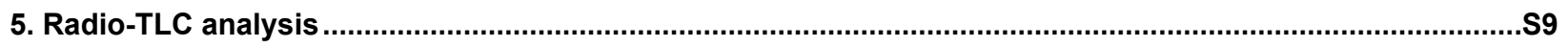

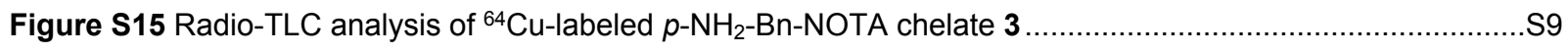

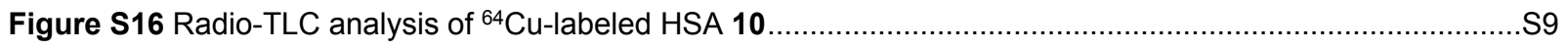

6. SDS-PAGE

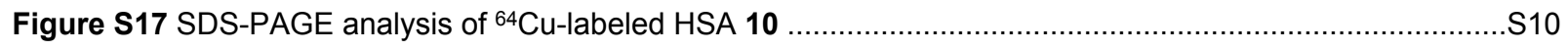

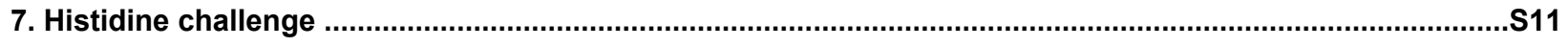

Figure S18 HPLC trace of histidine challenge experiment ........................................................................ 11

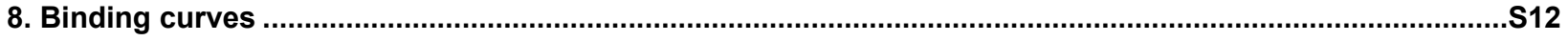

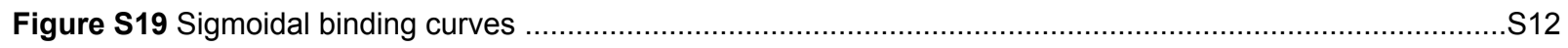

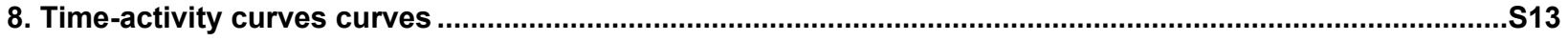

Figure S20 Time-activity curves of ${ }^{64} \mathrm{Cu}$-labeled HSA 10 over heart, liver, and kidneys ..................................S13 


\section{Reaction conditions using pre-conjugation versus post-conjugation labeling*}

Table S1. Reaction conditions tested for azo coupling of L-tyrosine with diazonium salt of $p-\mathrm{NH}_{2}-\mathrm{Bn}-\mathrm{NOTA}$.

\begin{tabular}{|c|c|c|c|}
\hline Entry & Solvent & $\mathrm{pH}$ & Result \\
\hline 1 & PBS & 6 & No product formation \\
\hline 2 & PBS & 8 & No product formation \\
\hline $3^{* *}$ & $\mathrm{HCl}: \mathrm{NaOH} 1: 1$ & 8 & No product formation \\
\hline 4 & $\mathrm{HCl}: \mathrm{NaOH} 1: 1$ & 10 & No product formation \\
\hline
\end{tabular}

Table S2. Reaction conditions tested for azo coupling of L-tyrosine with diazonium salt of 3-fluoroaniline.

\begin{tabular}{cccc}
\hline Entry & Solvent & pH & Result \\
\hline $\mathbf{1}$ & $\mathrm{HCl}: \mathrm{NaOH} 1: 1$ & 8 & Product detected \\
\hline $\mathbf{2}$ & $\mathrm{HCl}: \mathrm{NaOH} 1: 1$ & 10 & Product detected \\
\hline
\end{tabular}

Table S3. Reaction conditions tested for azo coupling of L-tyrosine with diazonium salt of natCu or natGalabeled $p$ - $\mathrm{NH}_{2}$-Bn-NOTA.

\begin{tabular}{ccccc}
\hline & Entry & Solvent & pH & Result \\
\hline${ }^{* *} \mathrm{n}=2$ & $\mathbf{1}^{* *}$ & HCl:PBS $(1: 1)$ & 9 & Product detected \\
\hline
\end{tabular}

Table S4. Reaction conditions tested for azo coupling of neurotensin NT(8-13) with diazonium salt of $p$ $\mathrm{NH}_{2}$-Bn-NOTA (entry 1). Reaction conditions tested for azo coupling of neurotensin NT(8-13) with diazonium salt of nat $\mathrm{Cu}$ or natGa-labeled $p-\mathrm{NH}_{2}-\mathrm{Bn}-\mathrm{NOTA}$ (entry 2 and 3 ).

\begin{tabular}{cccc}
\hline Entry & Solvent & pH & Result \\
\hline $\mathbf{1}$ & HCl:PBS $(1: 1)$ & $8-9$ & No product formation \\
\hline $\mathbf{2}$ & HCl:PBS $(1: 1)$ & $8-9$ & Product detected \\
\hline $\mathbf{3}$ & HCl:PBS $(1: 1)$ & $8-9$ & Product detected \\
\hline
\end{tabular}

${ }^{*}$ All reactions were analyzed with LC-MS. 


\section{LC-MS spectra}

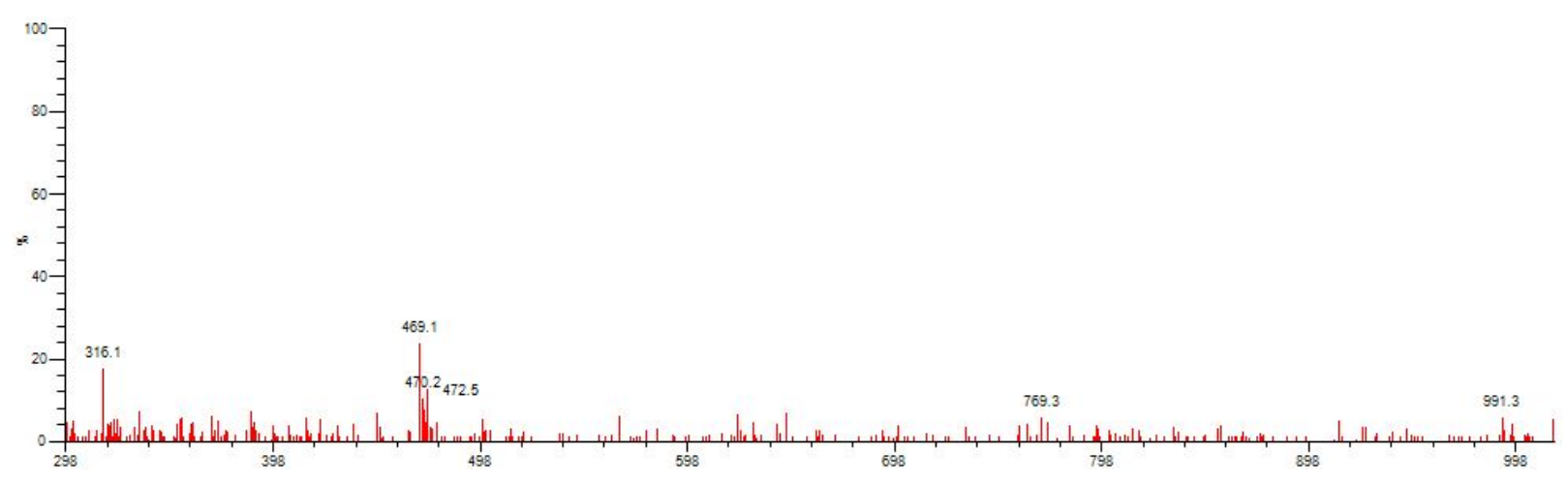

Figure S1. LC-MS spectrum of natCu-labeled $p-\mathrm{NH}_{2}-\mathrm{Bn}-\mathrm{NOTA}$ chelate. LC-MS, ESI-positive: 469.1 [M+H] ${ }^{+}$.

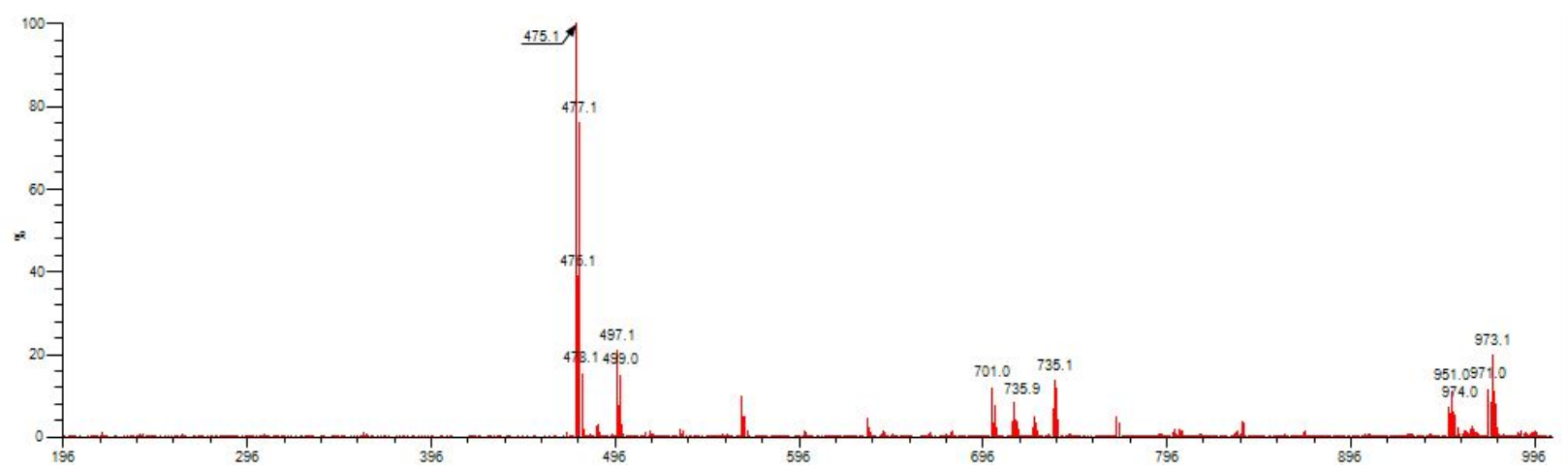

Figure S2. LC-MS spectrum of natGa-labeled $p-\mathrm{NH}_{2}-\mathrm{Bn}-\mathrm{NOTA}$ chelate. LC-MS, ESI-positive: 475.1 [M+H] ${ }^{+}$.

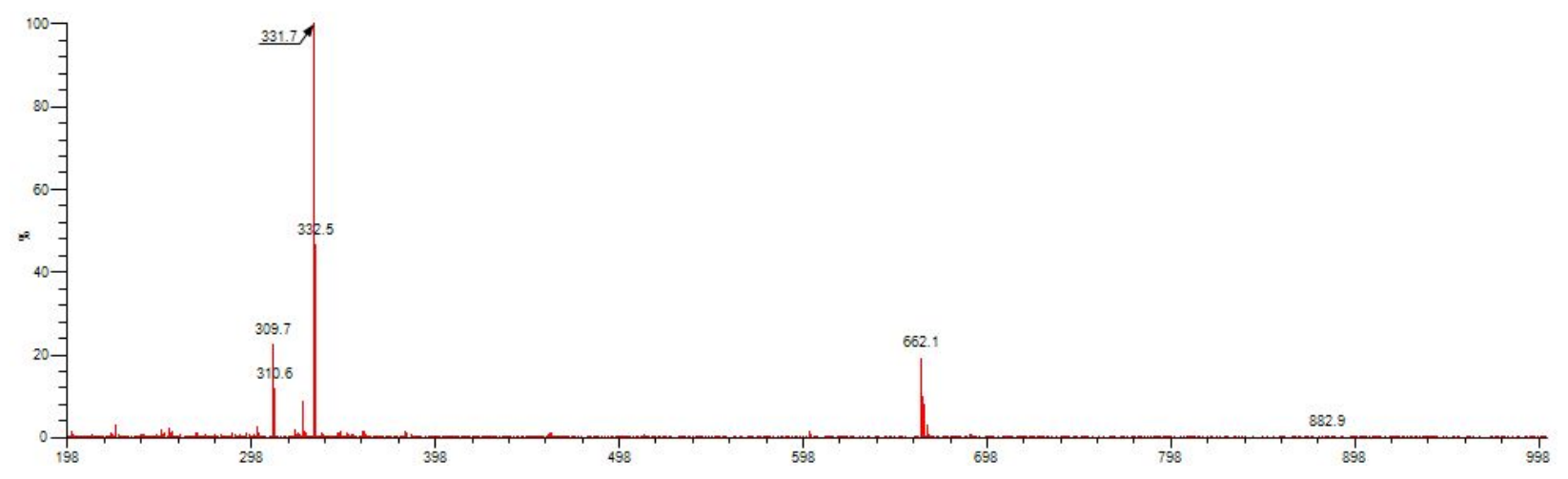

Figure S3. LC-MS spectrum of natCu-labeled L-tyrosine. LC-MS, ESI-positive: $662.1[\mathrm{M}+\mathrm{H}]^{+}$. 


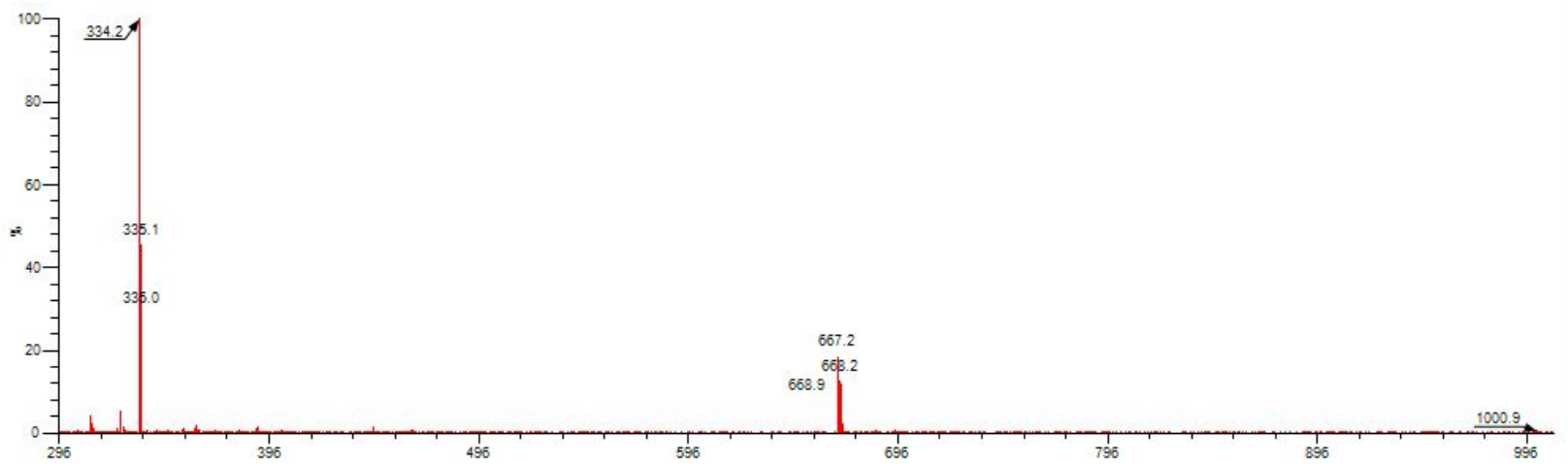

Figure S4. LC-MS spectrum of nat Ga-labeled L-tyrosine. LC-MS, ESI-positive: $667.2[\mathrm{M}+\mathrm{H}]^{+}$.

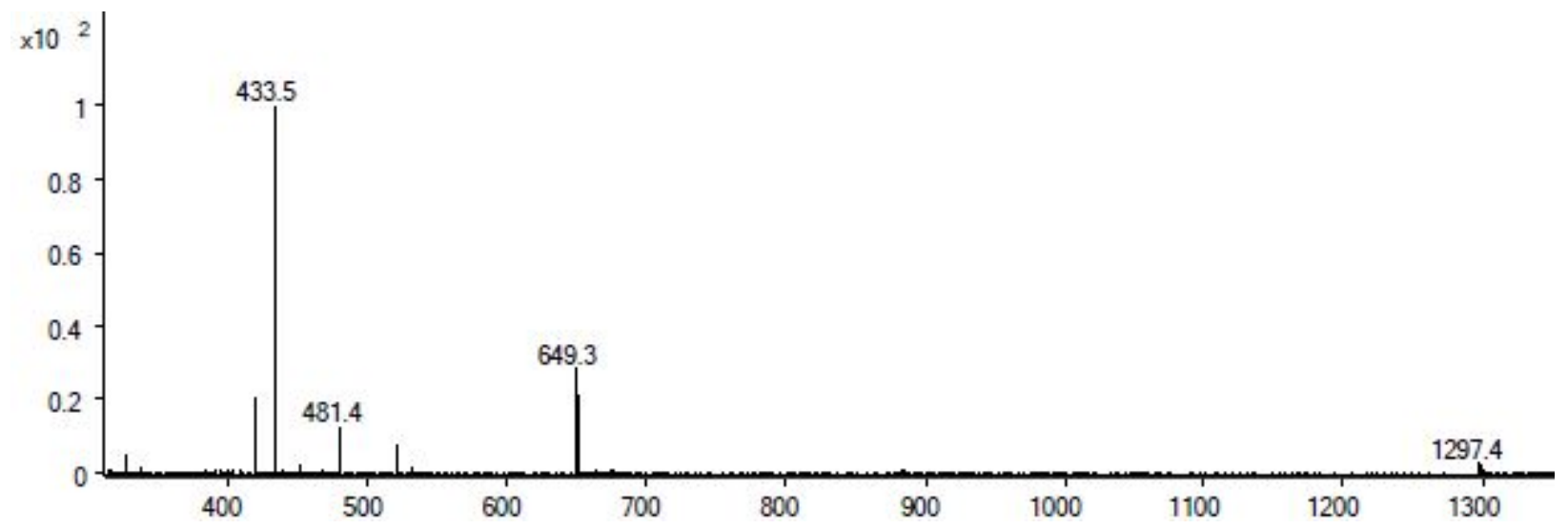

Figure S5. LC-MS spectrum of natCu-labeled neurotensin NT(8-13). LC-MS, ESI-positive: 1297.4 [M+H] ${ }^{+}$.

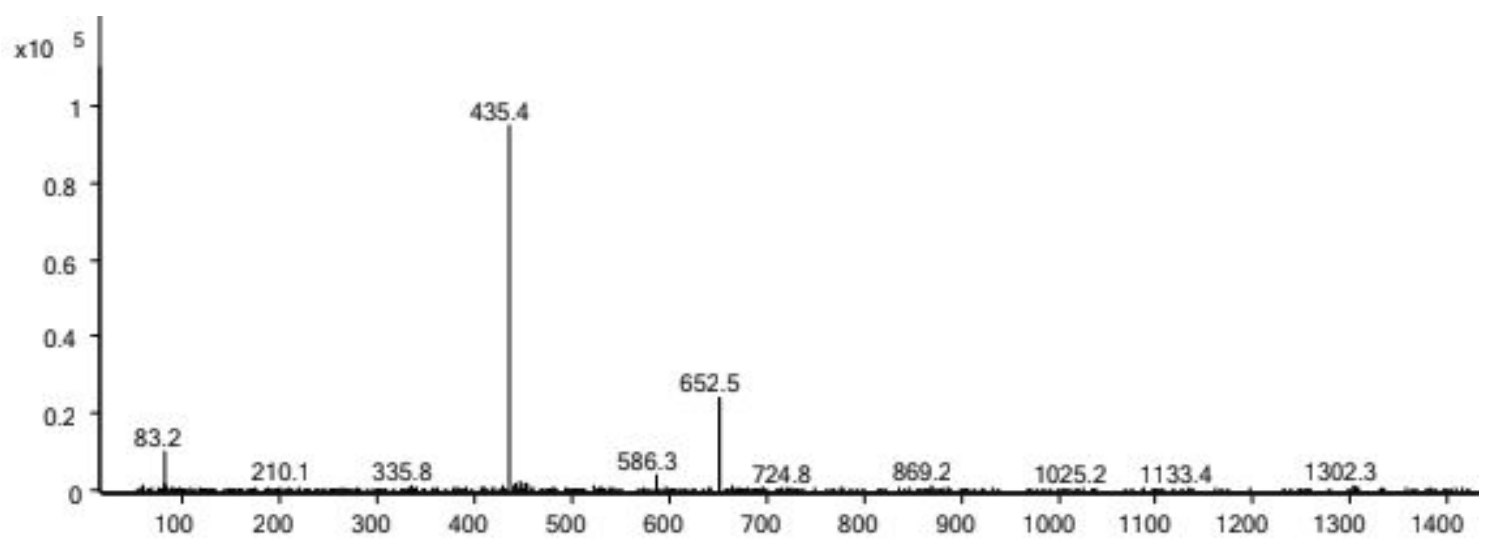

Figure S6. LC-MS spectrum of ${ }^{\text {nat }}$ Ga-labeled neurotensin NT(8-13). LC-MS, ESI-positive: 1302.3 [M+H] . 


\section{HPLC traces}

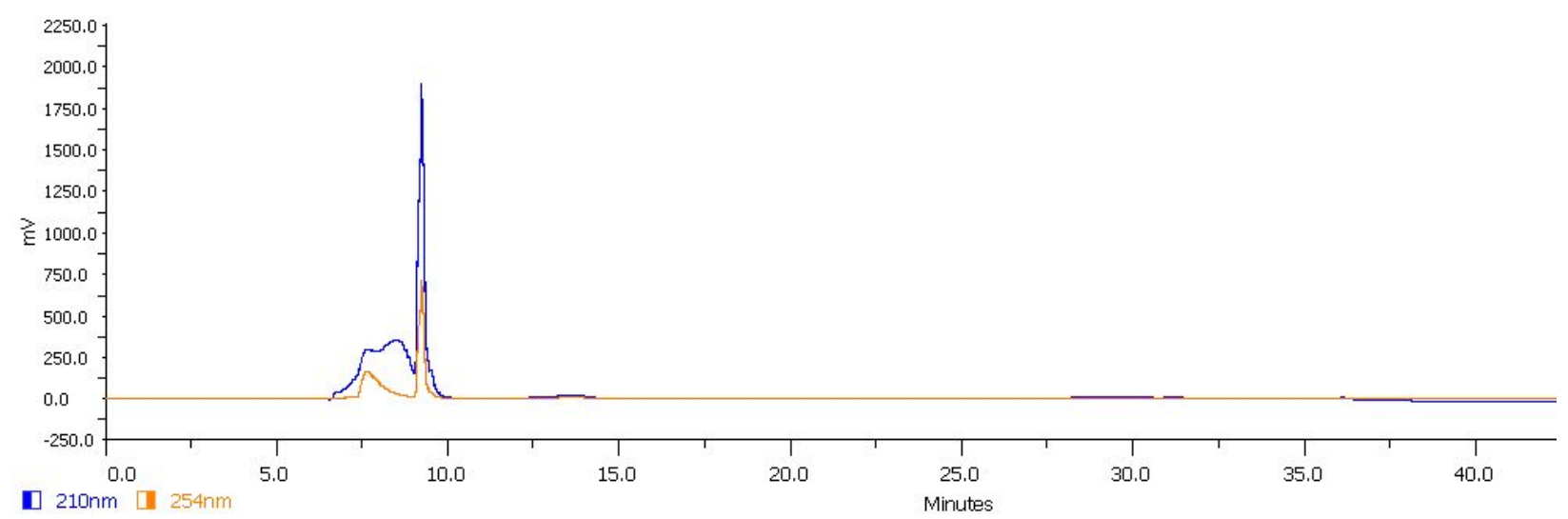

Figure S7. HPLC trace of natCu-labeled $p-\mathrm{NH}_{2}-\mathrm{Bn}-\mathrm{NOTA}$; retention time $9 \mathrm{~min}$.

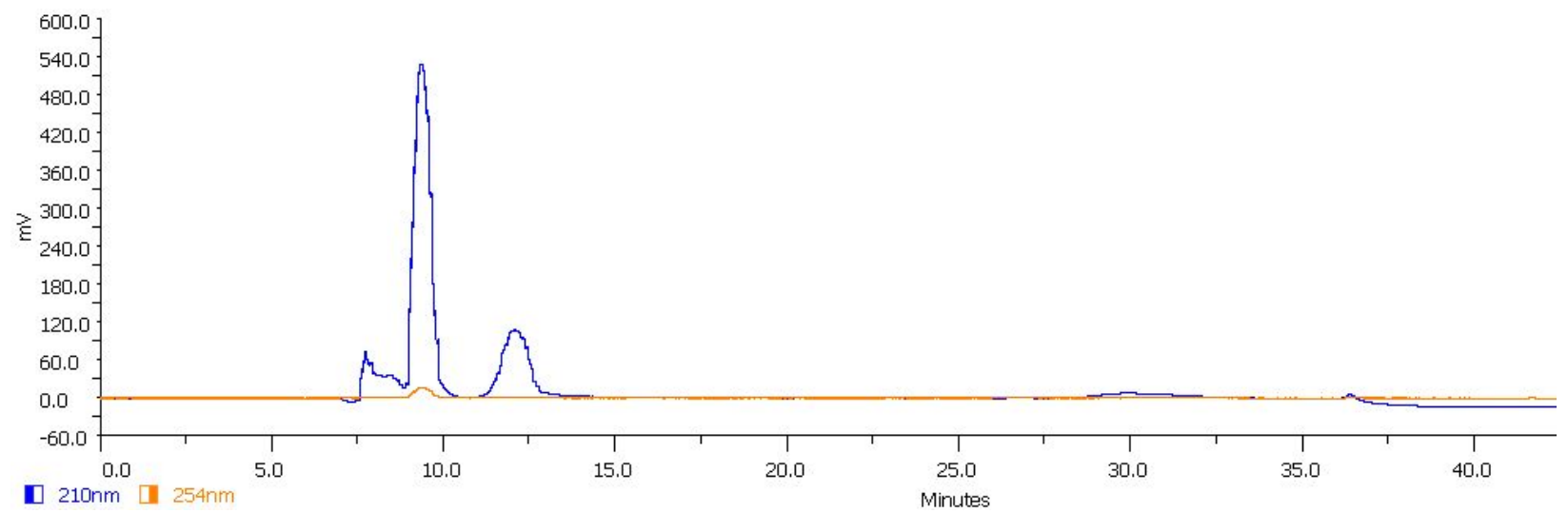

Figure S8. HPLC trace of nat Ga-labeled $p-\mathrm{NH}_{2}-\mathrm{Bn}-\mathrm{NOTA}$; retention time 9 min.

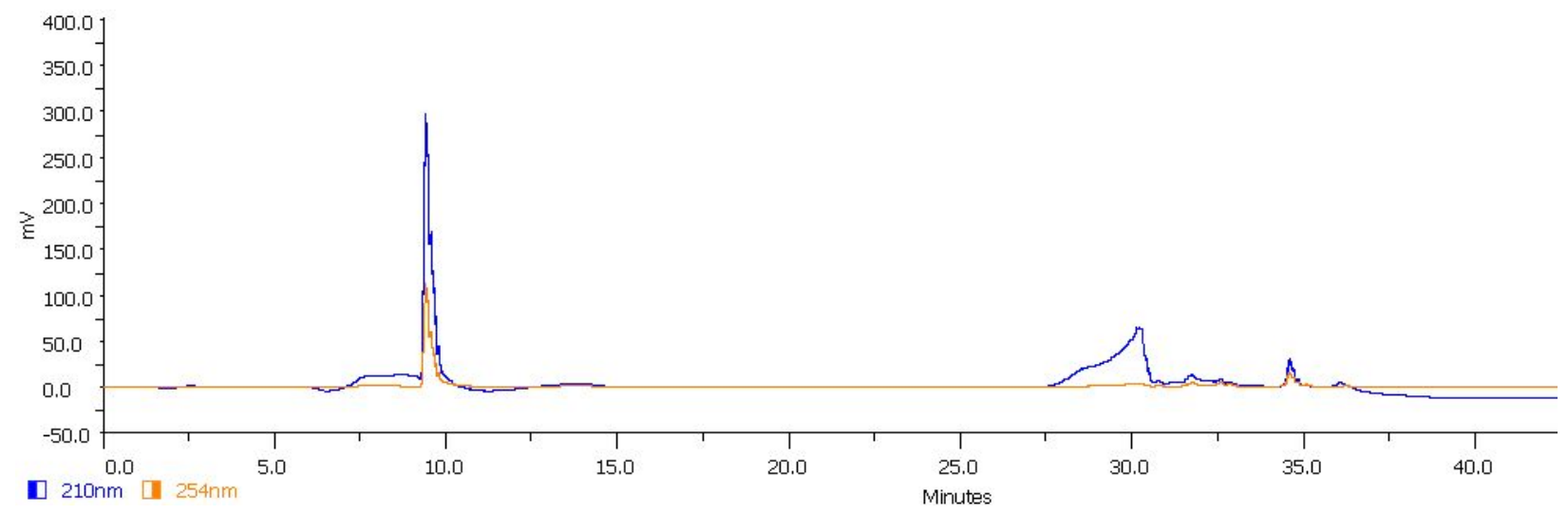

Figure S9. HPLC trace of ${ }^{\text {nat }} \mathrm{Cu}$-labeled L-tyrosine; retention time $34 \mathrm{~min}$. 


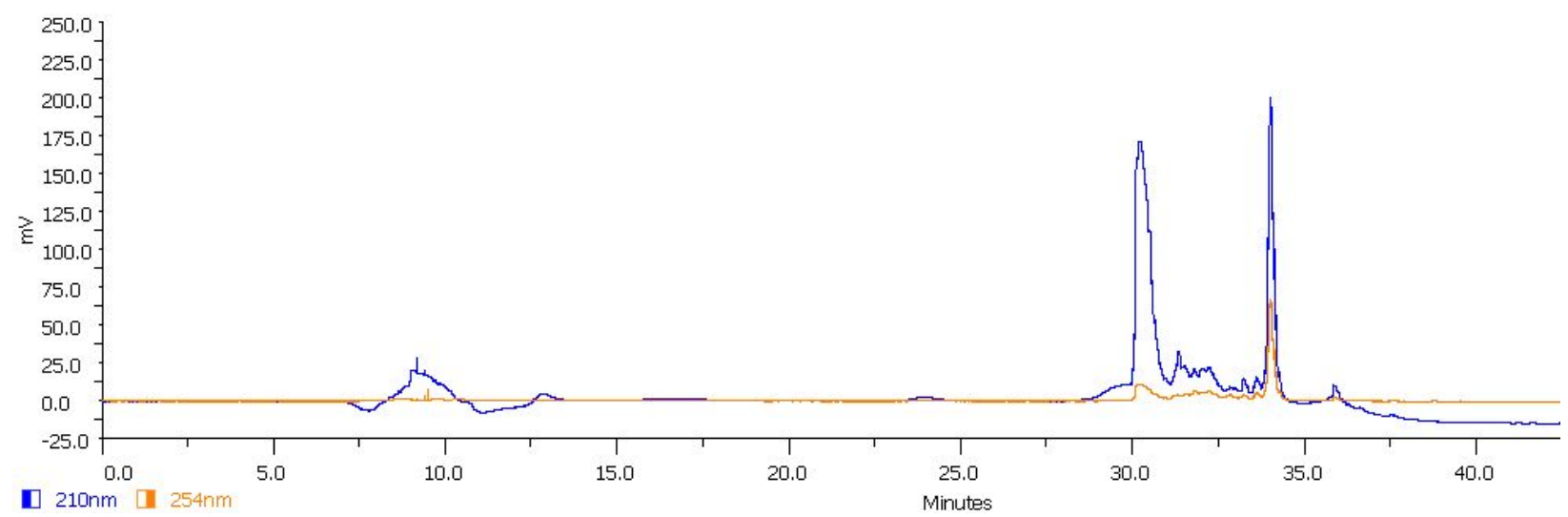

Figure S10. HPLC trace of natGa-labeled L-tyrosine; retention time 34 min.

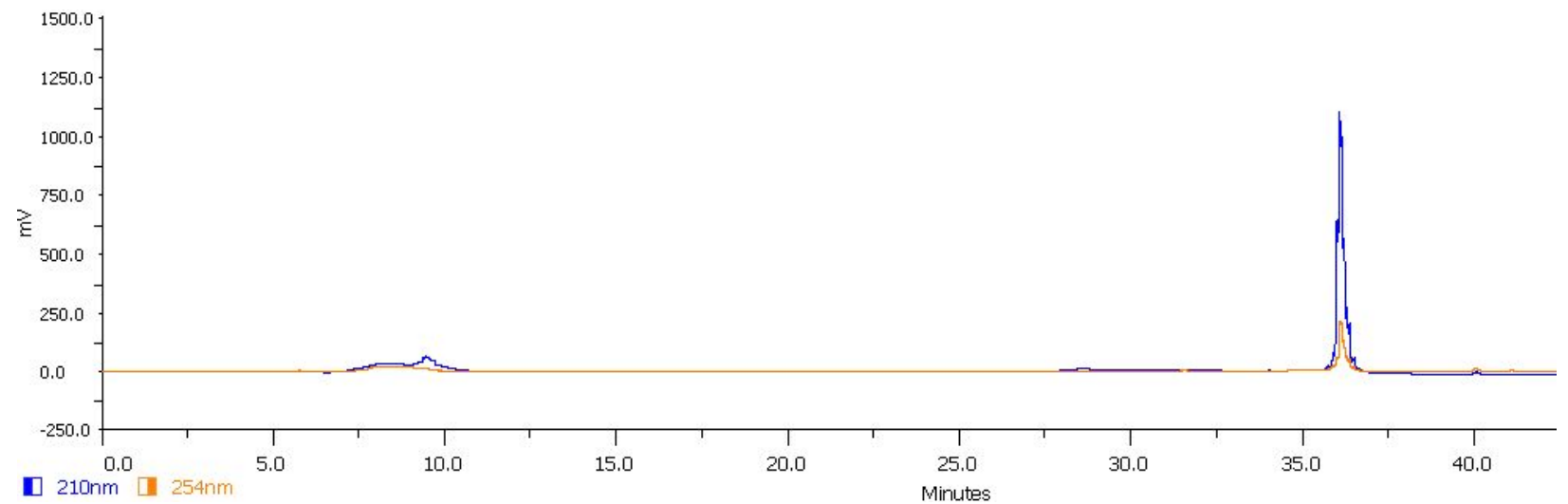

Figure S11. HPLC trace of natCu-labeled neurotensin NT(8-13); retention time 36 min.

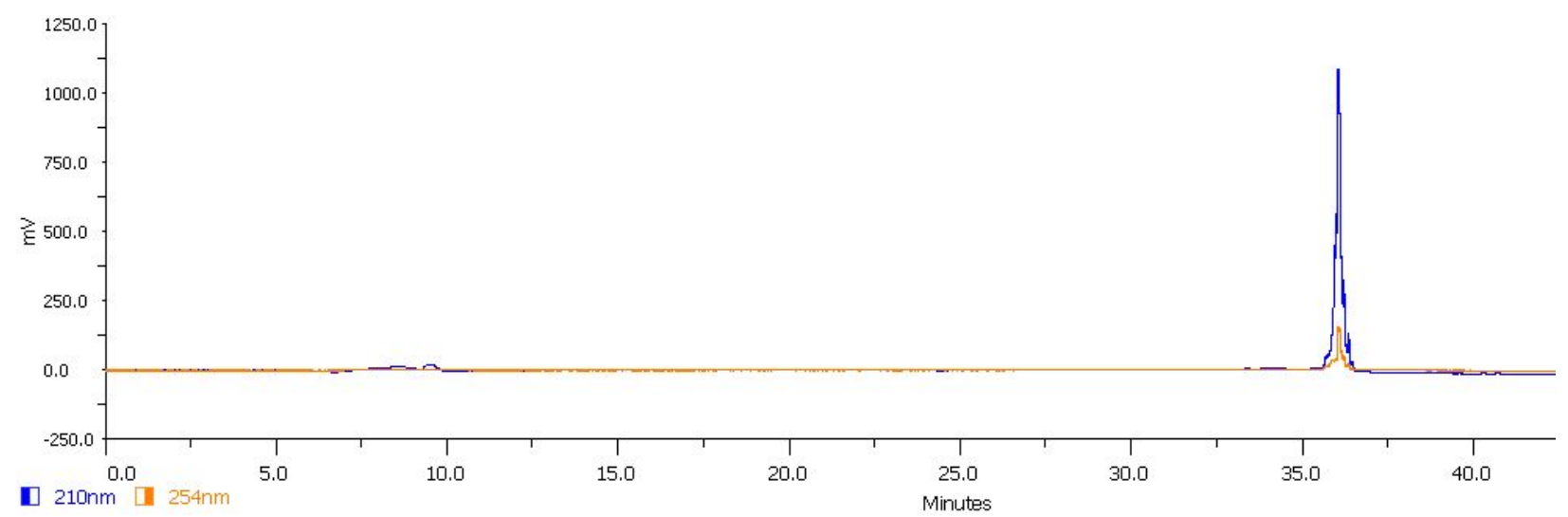

Figure S12. HPLC trace of nat Ga-labeled neurotensin NT(8-13); retention time 36 min. 


\section{Radio-HPLC traces}

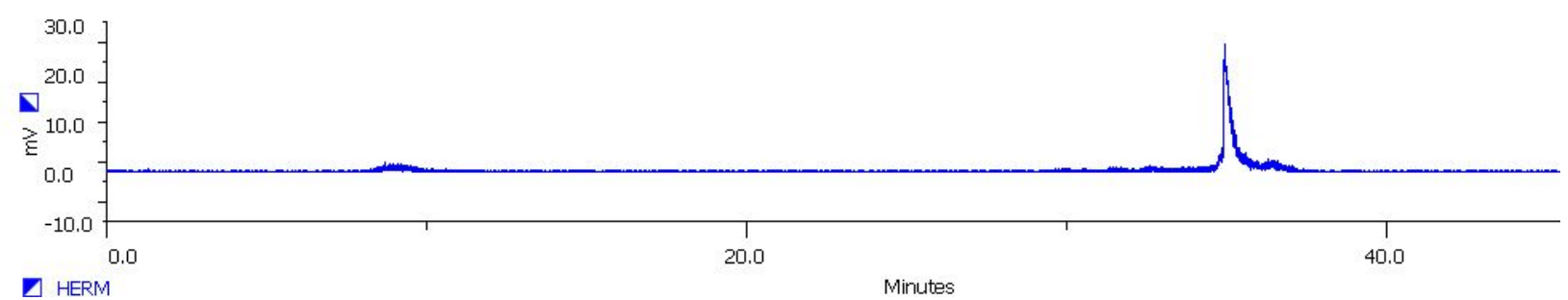

Figure S13. Radio-HPLC trace of ${ }^{64} \mathrm{Cu}$-labeled L-tyrosine; retention time $35 \mathrm{~min}$. Note that the same retention time was observed for ${ }^{68} \mathrm{Ga}$-labeled L-tyrosine.

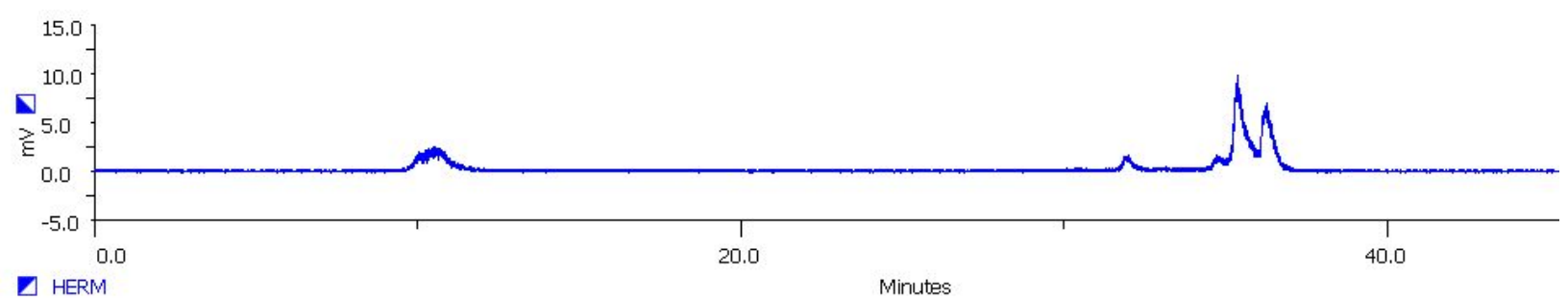

Figure S14. Radio-HPLC trace of ${ }^{64} \mathrm{Cu}$-labeled neurotensin NT(8-13); retention time $36 \mathrm{~min}$. Note that the same retention time was observed for ${ }^{68} \mathrm{Ga}$-labeled neurotensin $\mathrm{NT}(8-13)$. 


\section{Radio-TLC analysis}

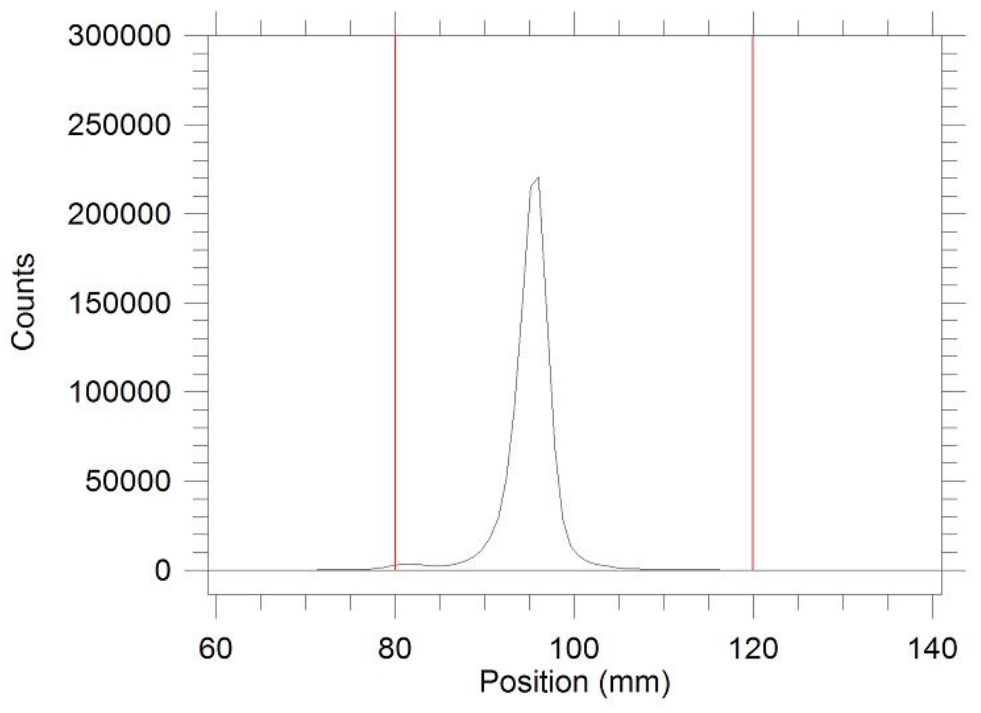

Figure S15. Radio-TLC analysis of ${ }^{64} \mathrm{Cu}$-labeled $p-\mathrm{NH}_{2}-\mathrm{Bn}-\mathrm{NOTA}$ chelate 3; reverse phase TLC plate and a $10 \% 1 \mathrm{M}$ ammonium acetate in methanol solvent system.

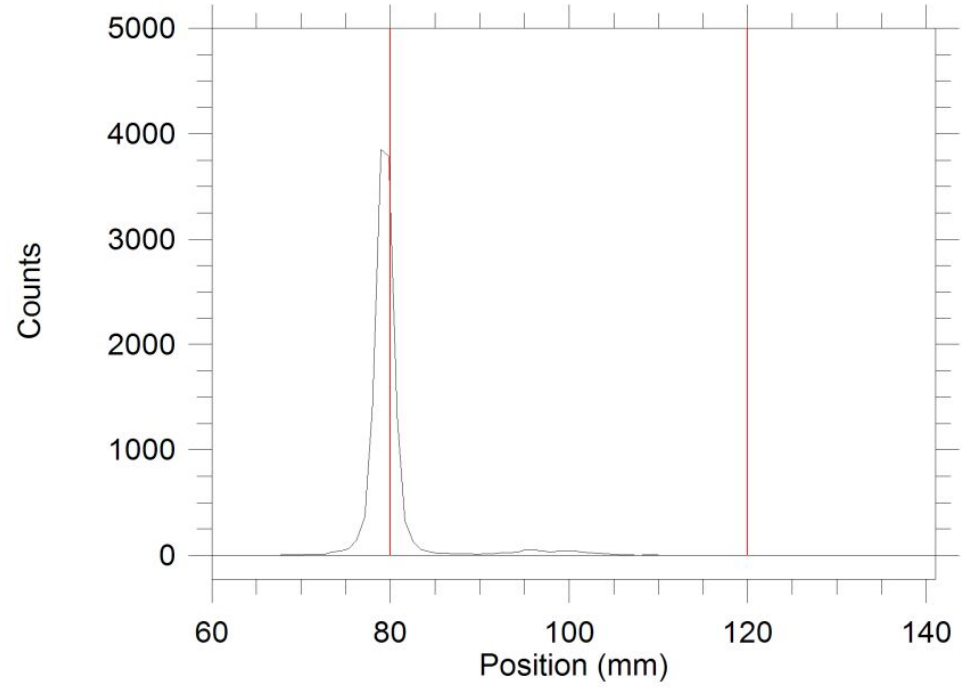

Figure S16. Radio-TLC analysis of ${ }^{64} \mathrm{Cu}$-labeled HSA 10; reverse phase TLC plate and a $10 \% 1 \mathrm{M}$ ammonium acetate in methanol solvent system. 


\section{SDS-PAGE analysis}

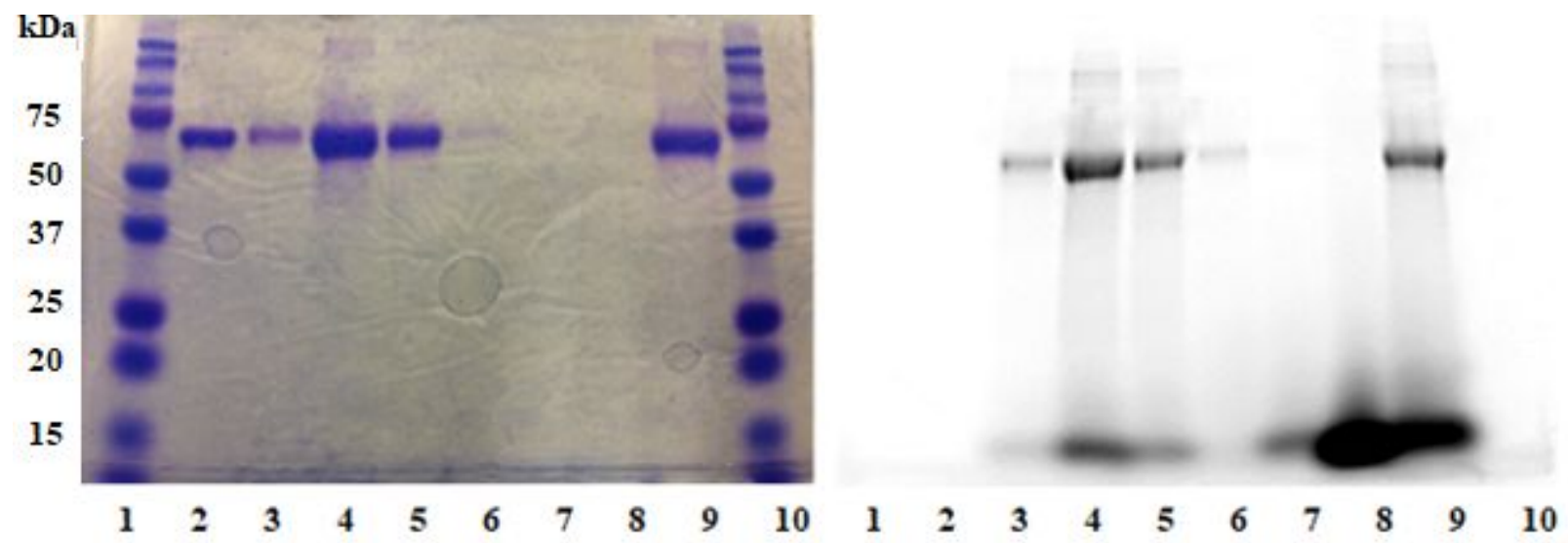

Figure S17. SDS-PAGE analysis of ${ }^{64} \mathrm{Cu}-\mathrm{HSA} 10$ with Coomassie stain (left) and phosphorimage (right). Lane 1 ladder, lane 2 non-radioactive HSA, lanes $3-6$ radiochemically pure fractions, lanes $7-8{ }^{64} \mathrm{Cu}-$ labeled $p-\mathrm{NH}_{2}$-Bn-NOTA, lane 9 crude radioactive reaction mixture, lane 10 ladder. 


\section{Histidine challenge}

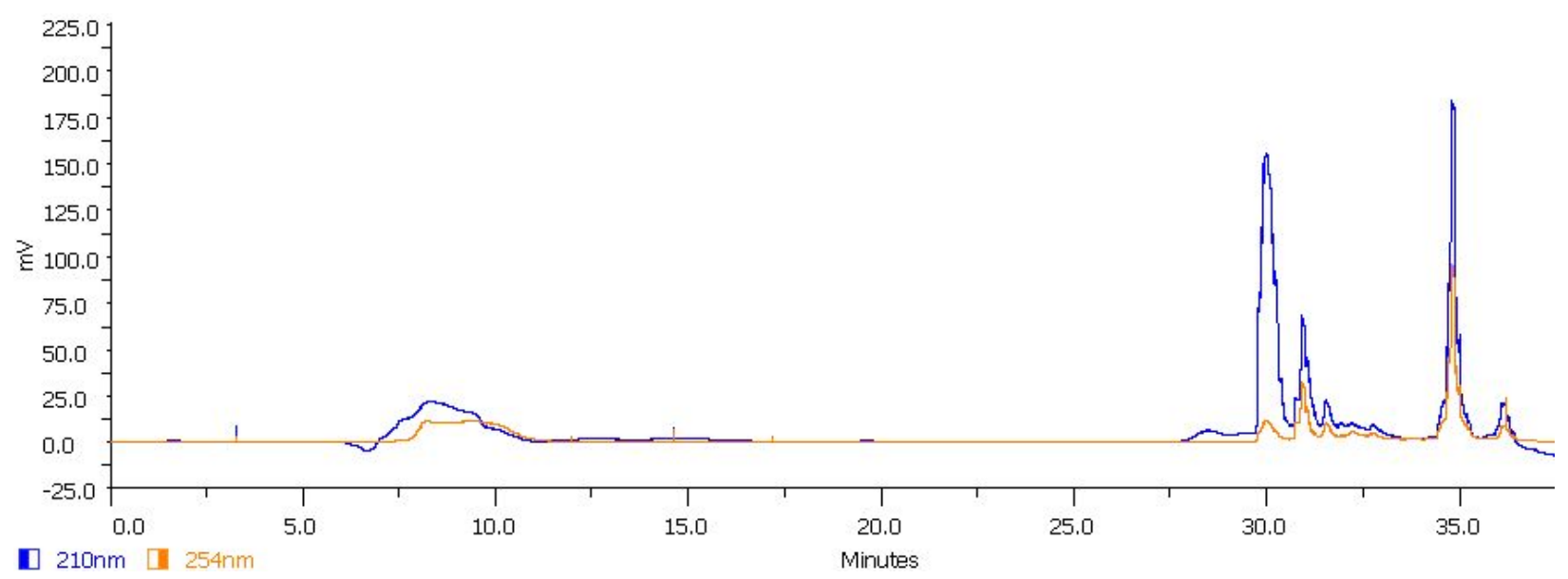

Figure S18. HPLC trace of histidine challenge experiment with ${ }^{\text {natC }}$-labeled diazonium salt 5 and equimolar amounts of L-tyrosine and L-histidine at $\mathrm{pH} \mathrm{9;} \mathrm{natCu-labeled} \mathrm{L-histidine} \mathrm{(retention} \mathrm{time} 30 \mathrm{~min}$ ) and natCu-labeled L-tyrosine (retention time $35 \mathrm{~min}$ ). 


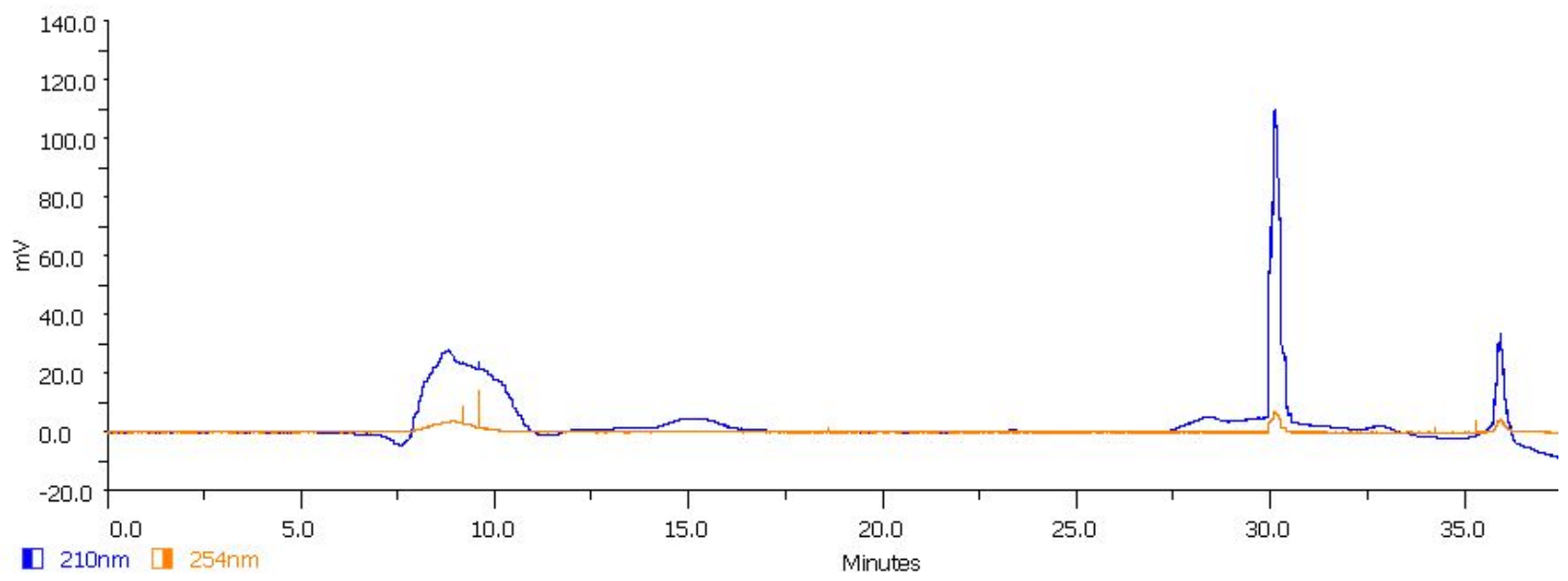

Figure S19. HPLC trace of histidine challenge experiment with ${ }^{\text {nat }} \mathrm{Cu}$-labeled diazonium salt $\mathbf{5}$ and equimolar amounts of L-tyrosine and L-histidine at $\mathrm{pH}$ 7; ${ }^{\text {nat }} \mathrm{Cu}$-labeled L-histidine (retention time $30 \mathrm{~min}$ ) and natCu-labeled L-tyrosine (retention time $35 \mathrm{~min}$ ). 


\section{Binding curves}

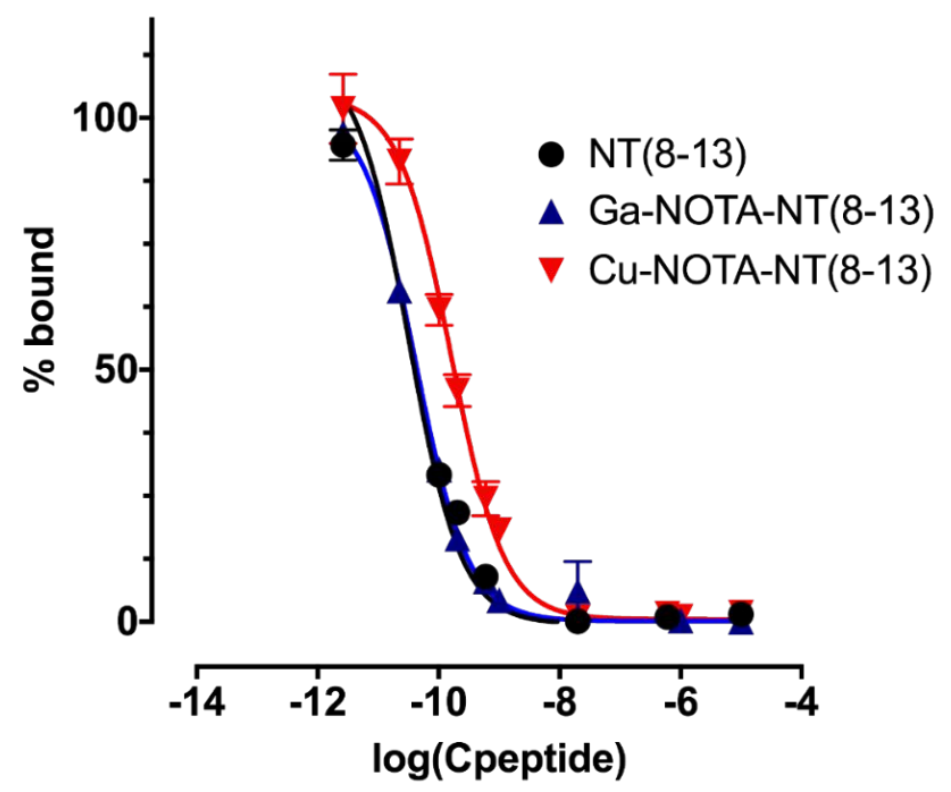

Figure S20. Sigmoidal binding curves of nat $\mathrm{Cu}$-labeled neurotensin NT(8-13) and ${ }^{\text {nat }} \mathrm{Ga-labeled}$ neurotensin $\mathrm{NT}(8-13)$ compared to unmodified neurotensin NT(8-13) $(n=3)$. 


\section{Time-activity curves}
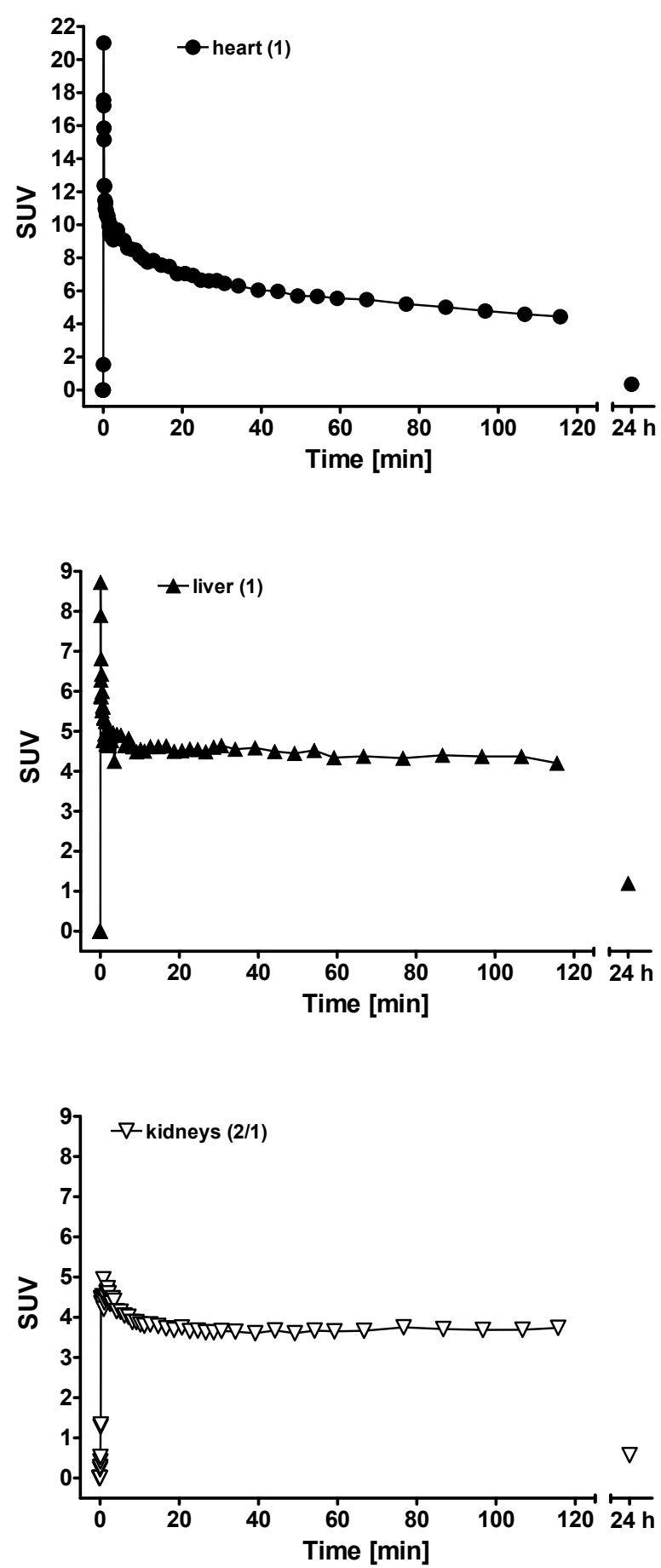

Figure S21. Time-activity curves of ${ }^{64} \mathrm{Cu}$-labeled HSA 10 over the heart, liver, and kidneys in normal BALB/C mice. 